\title{
Short-term gas dispersion in idealised urban canopy in street parallel with flow direction
}

\author{
Hana Chaloupecká ${ }^{1,2 a}$, Zbyněk Jaňour ${ }^{2}$ and Štěpán Nosek ${ }^{2}$ \\ ${ }^{1}$ Charles University in Prague, Faculty of Mathematics and Physics, Czech Republic \\ ${ }^{2}$ Institute of Thermomechanics Academy of Sciences of the Czech Republic, v.v.i. Dolejškova 1402/5, Prague 182 00, Czech Republic
}

\begin{abstract}
Chemical attacks (e.g. Syria 2014-15 chlorine, 2013 sarine or Iraq 2006-7 chlorine) as well as chemical plant disasters (e.g. Spain 2015 nitric oxide, ferric chloride; Texas 2014 methyl mercaptan) threaten mankind. In these crisis situations, gas clouds are released. Dispersion of gas clouds is the issue of interest investigated in this paper. The paper describes wind tunnel experiments of dispersion from ground level point gas source. The source is situated in a model of an idealised urban canopy. The short duration releases of passive contaminant ethane are created by an electromagnetic valve. The gas cloud concentrations are measured in individual places at the height of the human breathing zone within a street parallel with flow direction by Fast-response Ionisation Detector. The simulations of the gas release for each measurement position are repeated many times under the same experimental set up to obtain representative datasets. These datasets are analysed to compute puff characteristics (arrival, leaving time and duration). The results indicate that the mean value of the dimensionless arrival time can be described as a growing linear function of the dimensionless coordinate in the street parallel with flow direction where the gas source is situated. The same might be stated about the dimensionless leaving time as well as the dimensionless duration, however these fits are worse. Utilising a linear function, we might also estimate some other statistical characteristics from datasets than the datasets means (medians, trimeans). The datasets of the dimensionless arrival time, the dimensionless leaving time and the dimensionless duration can be fitted by the generalized extreme value distribution (GEV) in all sampling positions except one.
\end{abstract}

\section{Introduction}

The presented paper deals with short-term gas releases. Such releases are dominant in emergency situations. By way of illustration, this type of emergency situation occurred in Catalonia, which is in north-eastern Spain, in February 2015. From Catalonia chemical plant, toxic orange gas cloud spread into six municipalities in central Catalonia. The gas cloud released when contents of delivery (including nitric oxide and ferric chloride) were mixed. The cloud was disappearing only very slowly because of very little wind. More details about this disaster can be found in [1]. The duration of such leakages is usually less than one hour. Therefore, they do not belong to mean field. If we conduct an experiment of a gas release once, we can see only one scenario from many possible ones that would happen with the same experimental set-up. That is the reason why we must

\footnotetext{
a Hana Chaloupecká: hana.chaloupecka@it.cas.cz
}

repeat experiments many times to obtain statistically representative datasets. In this paper, we focus on dispersion from short-term gas releases in an urban area typical for European city inner parts.

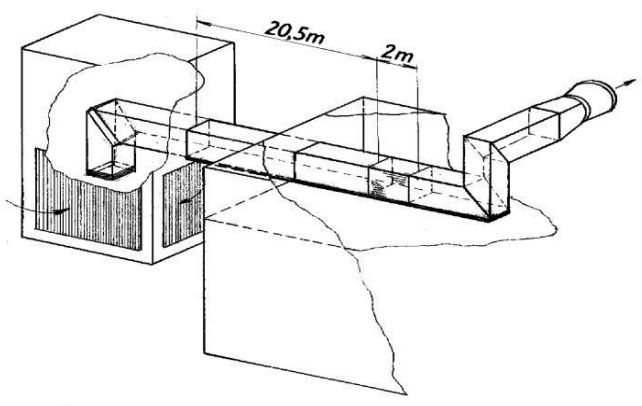

Figure 1. Scheme of an open low speed wind tunnel. 


\section{Experimental set-up}

The experiments are conducted in the Laboratory of Environmental Aerodynamics of the Institute of Thermomechanics, Academy of Sciences of the Czech Republic. This laboratory has an open low speed wind tunnel specialised in boundary layer modelling. The boundary layer is developed in $20.5 \mathrm{~m}$ long section (see Figure 1). For the boundary layer development with neutral stratification similar to the one found in cities, spires and roughness elements are employed. Behind this section, the incoming flow comes to a test section, where measurements are conducted. To the bottom of the test section, models are fixed.

The model in 1:400 manufacture scale used in the presented measurements represents an idealised urban area found in city centres in Europe. It consists of houses with pitched roofs $63 \mathrm{~mm}$ high $(50 \mathrm{~mm}$ houses bodies with $13 \mathrm{~mm}$ roofs). These houses are organised into closed courtyards of $150 \times 300 \mathrm{~mm}$ dimensions. The courtyards are placed equidistant from each other (50 $\mathrm{mm}$ ) in the model (see Figure 2).

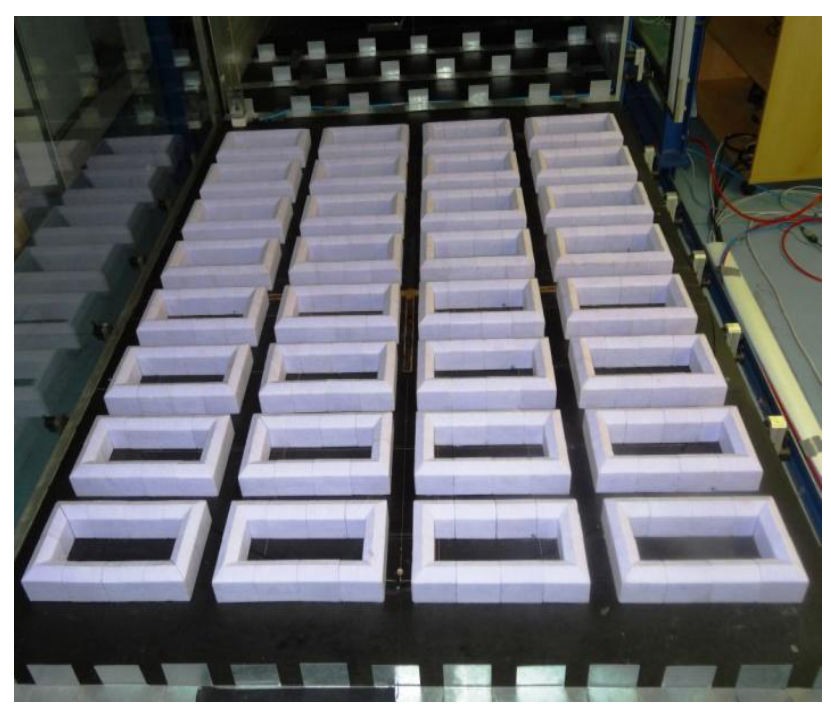

Figure 2. Model of an idealised urban canopy.

A ground level point gas source is simulated by a pipe. It is placed into a middle of a street parallel with the incoming flow direction in the model. The pipe is fasten into an electromagnetic valve. By this electromagnetic valve (3/2 electromagnetic valve of NORGREN) operated by a timer relay (Programmable Logic Controller Siemens LOGO!), short duration gas leakages are created (see [2] for more details). The leakages duration in the presented experiments is $1 \mathrm{~s}$ in the model scale (a little less than $7 \mathrm{~min}$ in reality).

Concentrations are measured by a fast flame ionisation detector (HFR400 fast flame ionisation detector of Cambustion, FFID). The FFID recorded concentration time series are smoothed to $6 \mathrm{~ms}$ averages in model scale (2.4 $\mathrm{s}$ in reality). The FFID capillary, which sucks in the mixture of air and tracer gas, is placed into individual sampling places at the model. To prevent its oscillations in flow, the capillary is protected by two outer capillaries. Further, the FFID chamber is covered by a wooden casing to prevent its cooling by ambient flow. Moreover, the FFID flexible cable, which connects the control unit and the sample head, is insulated against cooling down from ambient flow. The FFID capillary end is placed into the high corresponding to the human breathing zone during the experiments. As the tracer gas, we use ethane. The simulations of the gas release for each measurement position are repeated many times (about 400 times) under the same experimental set up to obtain representative datasets. This enables determination of mean gas cloud arrival time with approximately 2\%, mean cloud departure time with $4 \%$ and mean duration with $5 \%$ uncertainty at a sampling position. The uncertainties are counted from the repetitive measurements of 400 replicas at one measurement position.

\section{Data analysis}

In this paper, we present the results in dimensionless forms. To recalculate coordinates into the dimensionless form, we use the relation

$$
x^{*}=\frac{x}{H}, y^{*}=\frac{y}{H} \text {. }
$$

To recalculate the recorded concentration time series into the dimensionless form, we use the relation

$$
C^{*}=\frac{C U_{r e f} H^{2}}{Q} .
$$

This relation is valid for the point source. The tests of this dimensionless concentration values independence on the Reynolds number and on the source intensity were conducted to set the appropriate experimental conditions. To recalculate time into the dimensionless form, we use the relation

$$
t^{*}=\frac{t U_{r e f}}{H}
$$

In these relations $U_{\text {ref }}$ stands for the reference speed (correspondents to the free atmosphere), $\mathrm{H}$ the characteristic height (in this experiment houses height) and $\mathrm{Q}$ is the source intensity.

From the dimensionless concentration time series of each puff replica at each sampling place, puff characteristics are computed. The puff characteristics, which we discuss in this paper, are arrival time, leaving time and duration.

The data are analysed to obtain the puffs characteristics using the threshold criterion like in [3]. The threshold value is selected from the background concentration time series at individual sampling places. These time series are extracted from the recorded concentration time series of each puff replica (about 400 
at each sampling place) as the last few seconds of them. From these sequences, $99 \%$ percentiles of concentrations are calculated. Finally, we calculate the maximum of the founded $99 \%$ percentiles at each sampling place.

\subsection{Arrival (at*) \& leaving $\left(\right.$ lt $\left.^{*}\right)$ time}

Definition of puff arrival (departure) time using the threshold method combats a problem with undesirable spikes in concentration time series. The spikes can be recognised as sudden high concentration values in concentration time series. This increase affects only a few measured values. The described situation is usually caused by a dust particle that is sucked into the thin FFID capillary.

If the definition of the puff arrival time requires only detection of a value of at least the threshold quantity in concentration time series (searched from the time when gas is released) spikes can cause false identification of gas cloud arrival. This situation happens when the spikes are presented within the time interval between the gas release and the true searched time of a cloud arrival at a sampling place in concentration time series. A very similar situation can occur during an identification of a gas cloud departure.

As the spikes affect only a few measured values, the problem could be solved as proposed Doran et al. in [4]. They require the detection of at least the threshold value in a specified time interval instead of only one value. But the requirement of at least the threshold quantity detection in a whole specified interval in these time series in the definitions of cloud arrival (departure) is problematic because of a huge intermittency in concentration signals. Choosing too long time interval causes delayed detection of a cloud arrival at a sampling place or the cloud do not have to be detected at all. On the contrary if we choose too short time interval the spikes can be falsely analysed as a cloud arrival at a sampling place. Therefore we overcome this problem requirement of detection of the value of at least the threshold quantity not in the whole but only in a specified percentage of cases within a specified interval in concentration time series in the definitions like in [3]. Concretely we require the detection of the value of at least the threshold quantity in $50 \%$ values from $100 \mathrm{~ms}$ time interval.

\subsection{Duration (duration*)}

Duration of a puff in a sampling place can be defined by the relation

$$
\text { duration }=l t-a t \text {. }
$$

\section{Results}

The sampling places we present in this paper are chosen along the street parallel with flow direction (see Figure 3 ). The locations where the measurements took place are depicted by blue squares in Figure 3 . The cross represents the gas source position. The arrow marks the flow direction.

In this paper, we show a mean value from datasets at each investigated position. Moreover, we display box plots to graphically depiction of the datasets at each sampling position. In the box plots, a median value (depicted by a square), $25 \%-75 \%$ percentile (by a box) and a non-outlier range (by whiskers) are graphically represented. Furthermore, we display outliers (by circles) and extremes (by stars) in these figures. The outliers are defined as the values above (bellow) the $75 \%$ percentile ( $25 \%$ percentile) of the dataset plus (minus) 1.5 multiply of the difference between the $75 \%$ and $25 \%$ percentile. The extreme values are defined as the values above (bellow) the $75 \%$ percentile $(25 \%$ percentile) of the dataset plus (minus) 3 multiply of the difference between the $75 \%$ and $25 \%$ percentile. The scheme of this graphical representation is displayed in Figure 4.

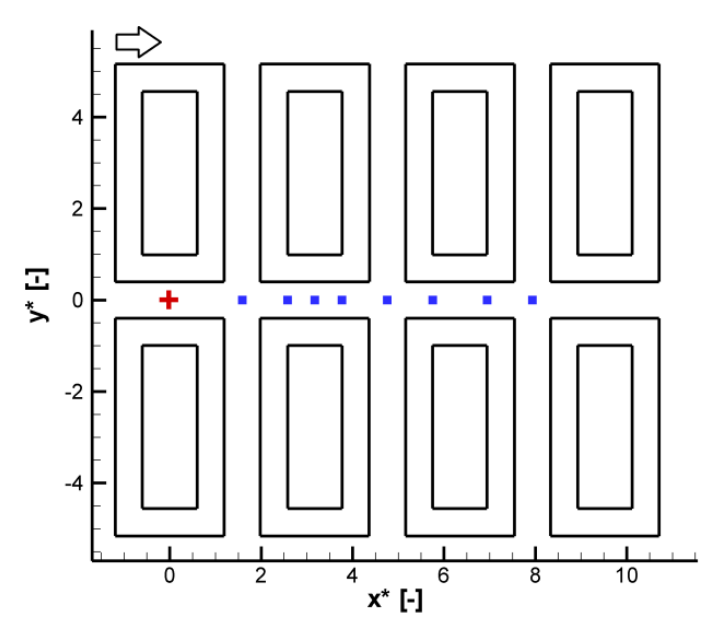

Figure 3. Scheme of investigated section.

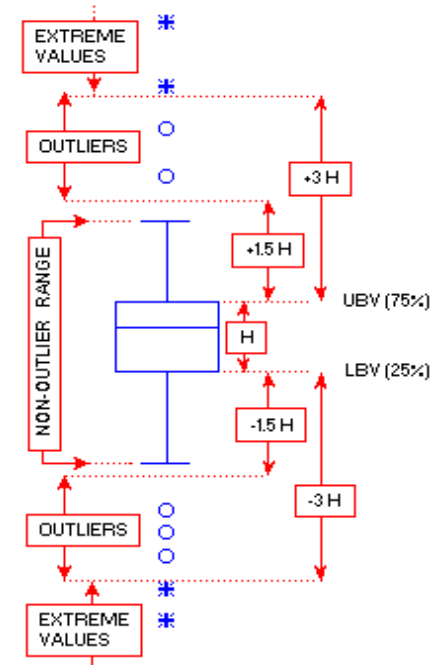

Figure 4. Scheme of graphical representation of datasets at each sampling place (adopted from [5]).

In Figure 5, dimensionless arrival time is displayed. The results indicate linear relationship between the 
dimensionless coordinate $\mathrm{x}$ and the mean value of the dimensionless arrival time. This relation can be described by the relation

$$
a t_{\text {mean }}^{*}=2.65( \pm 0.11) x^{*}+20.54( \pm 0.60) .
$$

It was found by the Least Squares approach. This linear model is proper according to an overall F-test, which tests a significance of regression, $(p=0.000001)$ as well as ttests testing a statistical significance of individual coefficients (intercept $p=0.000000$, independent value coefficient $p=0.000001)$. We also checked fulfilment of assumptions of the utilised method (independence of residuals and Gauss distribution of residuals with a constant mean value as well as variance). The found linear model has square of coefficient of determination $R^{2} 0.99$.

As well as the mean value of the dimensionless arrival time, also trimean of the dimensionless arrival time seems to be a linear function of the dimensionless coordinate $\mathrm{x}$. Utilising the same analysis method as in the mean value of the dimensionless arrival time, we found the equation

$$
a t_{\text {trimean }}^{*}=2.65( \pm 0.13) x^{*}+20.13( \pm 0.65) \text {. }
$$

The results of the statistical tests are very similar to those found in the mean value of the dimensionless arrival time. We checked fulfilment of assumptions of the utilised method as well. The square of the coefficient of determination $R^{2}$ is also 0.99 .

The datasets can be fitted by the generalized extreme value distribution (GEV) in all sampling positions except the location closest to the source. This hypothesis is not rejected according to the chi-square-goodness-of-fit test with alpha $=0.05$.

In Figure 6, the puff characteristic dimensionless leaving (departure) time is demonstrated. The precise assessment of cloud departure time from a sampling location is very difficult. The reason is that the cloud is very torn shortly before it leaves the sampling location as reported in [3]. In comparison with the dimensionless arrival time, the range of datasets is wider. Moreover, the datasets contain more extreme values. The results of the dimensionless leaving time indicate that a linear relationship might be found between the dimensionless coordinate $\mathrm{x}$ and the mean value of the dimensionless leaving time. The proposed model equation is

$$
l t_{\text {mean }}^{*}=14.03( \pm 2.74) x^{*}+164.55( \pm 13.71) \text {. }
$$

The equation (7) was found utilising the same approach as in the previous ones (F-test $p=0.002170$, t-tests: intercept $p=0.000020$, independent value coefficient $p=0.002170)$. The square of the coefficient of determination $R^{2}$ is 0.81 .

The median of the dimensionless leaving time might be also estimated as a linear function of the dimensionless coordinate $x$. If we use the median value instead of the mean, the square of the coefficient of determination becomes better -0.87 . Other relevant values are: F-test $p=0.000666$, t-tests: intercept $p=0.000006$, independent value coefficient $p=$ 0.000006 . The found equation is

$$
l t_{\text {median }}^{*}=13.53( \pm 2.10) x^{*}+156.43( \pm 10.52) \text {. }
$$

We could also use the trimean value of the dimensionless leaving time as in the equation (6).

$$
l t_{\text {trimean }}^{*}=13.69( \pm 2.59) x^{*}+162.60( \pm 12.99) \text {. }
$$

In this case the relevant values are: $R^{2}=0.82$, F-test $p=0.001875$, t-tests: intercept $p=0.000016$, independent value coefficient $p=0.001875$.

The datasets of the dimensionless leaving time can be fitted by GEV in all sampling positions except one $\left(x^{*} \approx 3.8\right)$. This hypothesis is not rejected according to the chi-square-goodness-of-fit test with alpha $=0.05$.

In Figure 7, the results of the dimensionless duration are depicted. In comparison with the equation (5) for the mean dimensionless arrival time, the line slope in the equation (7) for the dimensionless leaving time is higher. This denotes that the mean puff duration should increase with the increasing distance from the source along the the street parallel with flow direction. The described situation agrees with the results depicted in Figure 7. A linear function might be fitted to the data. However, this fit is worse than in all previous cases (equations (5) - (9)). The square of the coefficient of determination is only 0.73 . The proposed model is

$$
\begin{gathered}
\text { duration }_{\text {mean }}^{*}= \\
=11.38( \pm 2.85) x^{*}+144.01( \pm 14.26) .
\end{gathered}
$$

Other relevant data are: F-test $p=0.007167$, t-tests: intercept $p=0.000055$, independent value coefficient $p=0.007167$. This model can be also found utilising the equation (5) and (7) and the fact that duration= $l t-a t$.

The median of the dimensionless duration might be also described as a linear function of the dimensionless coordinate $\mathrm{x}$. This fact can be described by the relation

$$
\begin{array}{r}
\text { duration }_{\text {median }}^{*}= \\
=10.48( \pm 2.33) x^{*}+138.72( \pm 11.68) .
\end{array}
$$

Other relevant data are: F-test $p=0.004143$, t-tests: intercept $p=0.000022$, independent value coefficient $p=0.004143$. As well as in the dimensionless leaving time, this fit is better than in fitting the mean value because $R^{2}=0.77$.

A linear function might be also fitted between the trimean of the dimensionless duration and the dimensionless coordinate $x$. This situation can be described by the equation

$$
\begin{gathered}
\text { duration }_{\text {trimean }}^{*}= \\
=10.99( \pm 2.70) x^{*}+142.49( \pm 13.53) .
\end{gathered}
$$


Other relevant data are: F-test $p=0.006613$, t-tests: intercept $p=0.000043$, independent value coefficient $p=0.006613, R^{2}=0.73$.

The datasets of the dimensionless duration can be fitted by GEV in all sampling positions except one $\left(x^{*} \approx 3.8\right)$. This hypothesis is not rejected according to the chi-square-goodness-of-fit test with alpha $=0.05$. This is exactly the same as in the dimensionless leaving time.

\section{Conclusions}

This paper deals with short-term gas releases (puffs). The puff characteristics definition of arrival and leaving time are crucial in the data analysis. We define them using the threshold criterion. These definitions must cope with the undesirable spikes in concentration time signals as well as the huge signal intermittency. We overcome this problem requirement of detection of the value of at least the threshold quantity not in the whole but only in a specified percentage of cases within a specified interval in concentration time series in the definitions of arrival (leaving) time.

The mean value of the dimensionless arrival time can be described as a growing linear function of the dimensionless coordinate $\mathrm{x}$ in the street with the gas source parallel with flow direction by the relation $a t_{\text {mean }}^{*}=2.65( \pm 0.11) x^{*}+20.54( \pm 0.60), R^{2}=0.99$. A similar relation might be written for the mean value of the dimensionless leaving time, but the fit is worse $\left(R^{2}=0.81\right)$. This fact can cohere with the difficult assessment of the precise departure time (discontinuity of the gas cloud tail). The proposed model is $l t_{\text {mean }}^{*}=$ $14.03( \pm 2.74) x^{*}+164.55( \pm 13.71)$. In comparison with the equation for the mean dimensionless arrival time, the line slope is in the dimensionless leaving time higher. This denotes that the mean puff duration should increase with the increasing distance from the source along the the street parallel with flow direction. The described situation agrees with the found results. A linear function might be also fitted to the data. The proposed model is duration mean $^{*}=11.38( \pm 2.85) x^{*}+$ $144.01( \pm 14.26)$, but $R^{2}=0.73$. This model can be also found utilising the equation for the mean value of the dimensionless arrival time and the mean value of the dimensionless leaving time and the fact that duration $=$ lt -at. Utilising a linear function, we might also estimate some other statistical characteristics from datasets than the means (medians, trimeans).

The datasets of the dimensionless arrival time, the dimensionless leaving time and the dimensionless duration can be fitted by GEV in all sampling positions except one. This hypothesis is not rejected according to the chi-square-goodness-of-fit test with alpha $=0.05$

\section{Acknowledgement}

Authors thank for support from the grant LD12007 of the Czech Ministry of Education and Sport and the grant $15-$ 1894S of the Czech Science Foundation.

\section{References}

1. Toxic orange cloud spreads over Catalonia after chemical blast, http://www.theguardian.com/

2. H. Chaloupecká, Z. Jaňour, K. Jurčáková, L. Kukačka, Š. Nosek, EPJ Web of Conferences 67, 020 45 (2014)

3. H. Chaloupecká, Z. Jaňour. $9^{\text {th }}$ International Conference on Urban Climate jointly with $12^{\text {th }}$ Symposium on the Urban Environment (2015).

4. J.C. Doran, K.J. Allwine, J.E. Flaherty, K.L. Clawson, R.G. Carter. Atmospheric Environment 41, 3440-3452 (2007).

5. STATISTICA Help, http://documentation.statsoft.com

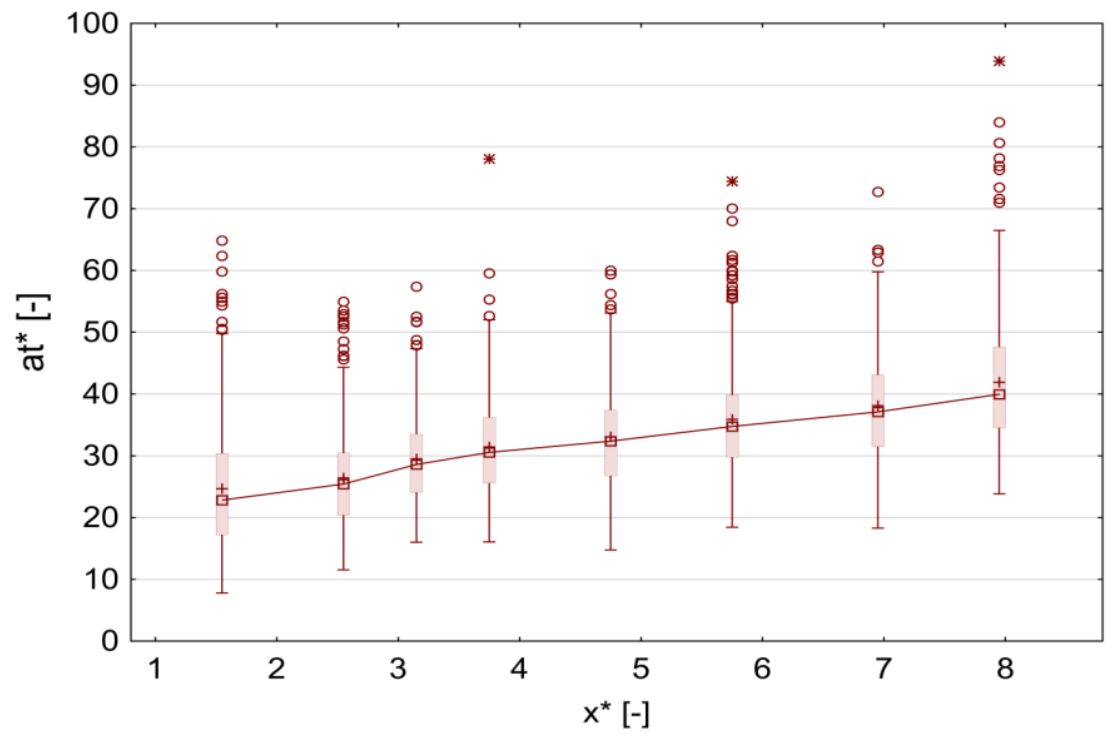

Figure 5. Dimensionless arrival time. 


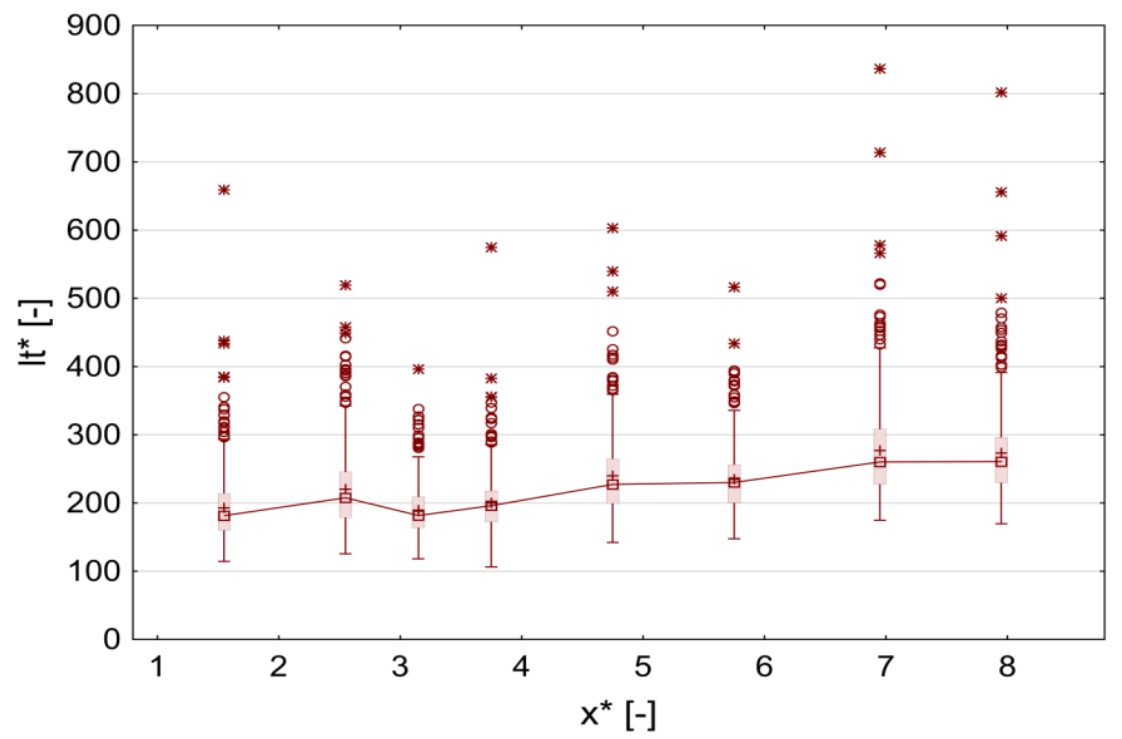

Figure 6. Dimensionless leaving time.

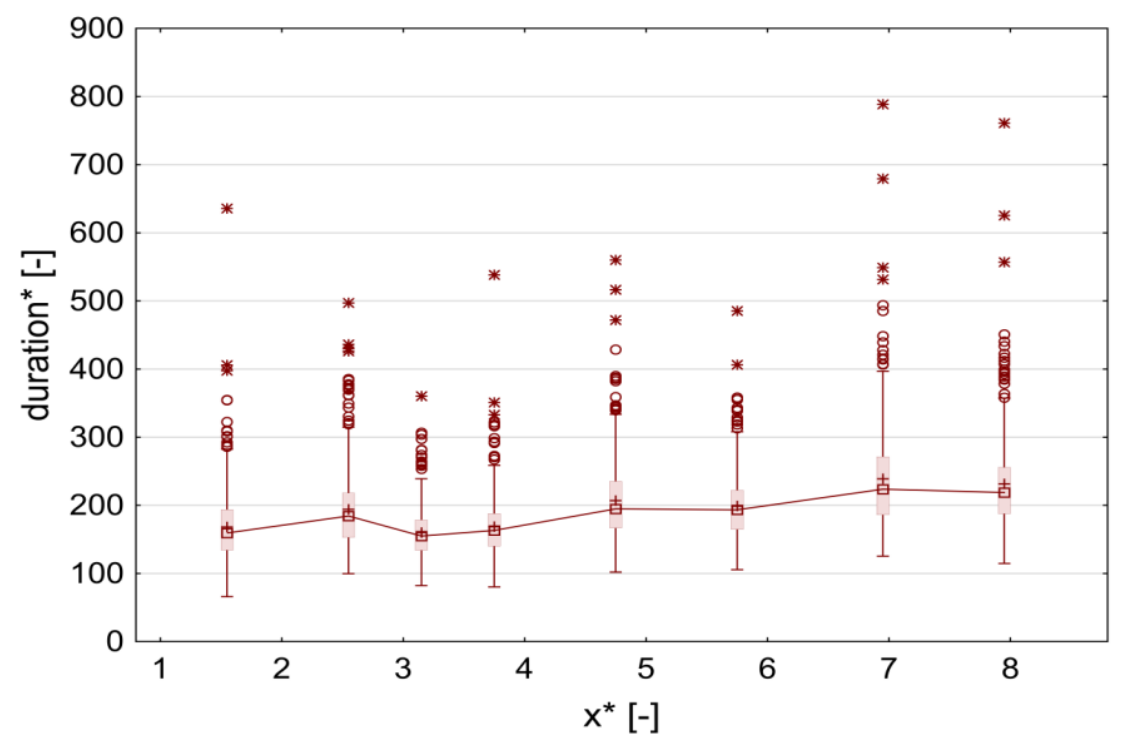

Figure 7. Dimensionless duration. 